rh 19 Revue d'histoire du XIXe siècle

Société d'histoire de la révolution de 1848 et des

révolutions du XIXe siècle

$24 \mid 2002$

Varia

Martyn LYONS, Readers and Society in Nineteenth-

Century France. Workers, Women, Peasants

Houndsmill, Palgrave, 2001, 208 p.

Philippe Chassaigne

\title{
OpenEdition
}

Journals

Édition électronique

URL : http://journals.openedition.org/rh19/388

DOI : $10.4000 /$ rh 19.388

ISSN : $1777-5329$

Éditeur

La Société de 1848

Édition imprimée

Date de publication : 1 juin 2002

Pagination : 188-190

ISSN : 1265-1354

Référence électronique

Philippe Chassaigne, « Martyn LYONS, Readers and Society in Nineteenth-Century France. Workers, Women, Peasants », Revue d'histoire du XIXe siècle [En ligne], 24 | 2002, mis en ligne le 28 juin 2005, consulté le 22 septembre 2020. URL : http://journals.openedition.org/rh19/388 ; DOI : https://doi.org/ $10.4000 /$ rh19.388

Ce document a été généré automatiquement le 22 septembre 2020.

Tous droits réservés 


\section{Martyn LYONS, Readers and Society in Nineteenth-Century France. Workers, Women, Peasants}

Houndsmill, Palgrave, 2001, 208 p.

Philippe Chassaigne

1 Professeur à l'université de Nouvelles Galles du Sud (Australie), Martyn Lyons est, aux côtés de Roger Chartier ou de Jean-Yves Mollier, l'un des meilleurs historiens du livre, au moins connu du public français pour son Triomphe du livre, publié directement dans notre langue ${ }^{1}$. Readers and Society traite de la massification de la diffusion du livre et de la lecture, qui est l'une des caractéristiques majeures de l'histoire culturelle du XIX à travers deux aspects précis : d'une part, les inquiétudes suscitées par l'accès à la lecture de nouvelles catégories sociales --ici, les femmes, les ouvriers et les paysans-- auprès des classes moyennes et des élites, et les mesures prises par pour canaliser ces comportements nouveaux, susceptibles de favoriser la propagation des "mauvaises idées" dans des publics inexpérimentés et supposés facilement influençables; d'autre part, les usages et comportements propres à ces nouveaux lecteurs --qui lit, que et comment lit-on ?-- et le possible décalage entre les ambitions de ceux-là et les pratiques de ceux-ci. Là se situe l'articulation essentielle de l'ouvrage.

Dans le cas des ouvriers, la nature de leurs lectures fait partie intégrante de la "question sociale": catholiques et républicains s'alarment du succès de la littérature populaire bon marché (littérature de colportage, romans feuilletons, journaux à un sou, etc.), les premiers pour leur effet démoralisateur, les seconds parce qu'ils concourent à l'aliénation politique de la classe ouvrière --un point de vue d'ailleurs partagé aussi par un certain nombre d'autodidactes issus du monde ouvrier. Cette identité de diagnostic pouvait conduire à l'identité des mesures adoptées, avec la création de bibliothèques de prêt d'obédiences certes diverses, mais dans lesquelles les ouvrages étaient soigneusement sélectionnés : ouvrages édifiants pour les catholiques, "classiques" (de Plutarque à Shakespeare en passant par Fénelon ou Molière) pour des institutions comme la Société Franklin ou la Ligue de l'enseignement. Toutefois, les travailleurs se 
montraient eux-mêmes très faiblement réceptifs à ces influences extérieures. Les recherches de Lyons font ressortir de profondes similitudes avec les comportements des ouvriers britanniques en quête de self improvement, tels qu'a pu les décrire le Britannique David Vincent: on retrouve la véritable boulimie de lectures caractéristique de l'ouvrier autodidacte, sa préférence pour les lectures utiles plutôt que pour les romans, ou encore le passage quasiment naturel de la lecture à la production littéraire propre, dont la fonction émancipatrice et identitaire ressort avec netteté. En revanche, ceux qui lisent de façon plus occasionnelle manifestent un goût prononcé pour la littérature d'évasion : les romans représentent plus de la moitié des ouvrages empruntés dans les bibliothèques municipales de Paris dans les deux dernières décennies du siècle, détail suffisamment révélateur à lui seul quant à l'impact réel des tentatives de "contrôle social", ou plutôt, ici, culturel, en tout genre.

Les femmes constituent le deuxième groupe des "nouveaux lecteurs" du XIX siècle, même si, Lyons en convient dès le début du livre, la nouveauté repose d'abord sur leur émergence en tant que segment de marché distinct: de fait, les femmes, du moins celles qui appartenaient aux classes moyennes, et plus encore aux classes moyennes provinciales, paraissent comme de grosses consommatrices de livres. Dans leur cas, les craintes ressortissent moins à leur appartenance sociale qu'aux conceptions -masculines-- en vigueur sur la psychologie de leur sexe et sur les conséquences envisageables de leur goût prononcé pour les romans sentimentaux, accusés de pervertir leurs sens et de dévoyer leur imagination : toutes les lectrices de romans font figure de "Madames Bovary" en puissance. Là encore, les "modèles positifs" proposés émanent de deux milieux diamétralement opposé, catholiques d'abord, féministes ensuite. Les premiers, très soucieux des femmes puisqu'elles constituent alors, on le sait, un élément clef de leur dispositif stratégique de résistance à la déchristianisation, prônent une lectrice attentive, réfléchie et critique, dédaigneuse des romans au profit de lectures instructives : les œuvres recommandées sont certes toujours des classiques et non des publications contemporaines, et on ne trouve nul encouragement à se frotter de positivisme ou de darwinisme, mais Lyons, comme bon nombre d'historiens du catholicisme avant lui (Langlois, Cholvy...), insiste sur le fait que ces préceptes ont contribué à élargir effectivement l'horizon intellectuel des femmes. Quant au modèle féministe, qui s'affirme à la fin du XIX ${ }^{e}$ siècle avec l'importation en France du modèle de la "nouvelle femme" venu des pays anglo-saxons, il n'est finalement pas si éloigné du modèle catholique dans ses principes de base: si, par exemple, en 1906, une rédactrice du bimensuel La Femme nouvelle, alors publié par Fernand Nathan, préconise un programme de lecture sensiblement plus axé sur les auteurs contemporains, associant Corneille, Racine et Pascal à Quinet, Byron, Michelet, Chateaubriand ou Madame de Staël, il s'en rapproche néanmoins par son souci d'encadrer les lectures des jeunes filles, puis des jeunes femmes, et de les détourner des "lectures hasardeuses". À l'opposée de ces tentatives, la pratique de la lecture pour les femmes, comme pour les ouvriers, participe bien à la définition d'un espace social qui leur soit propre. Lyons, en s'appuyant sur un intéressant corpus de textes autobiographiques (tel le Journal d'Eugénie de Guérin, datant des années 1830, ou la correspondance d'Hélène Legros, dans les années 1890, les Mémoires de Louise Weiss, etc.), tente d'en définir les contenus et la pratique: la place importante occupée par les romans, nonobstant les recommandations contraires des uns ou des autres, semble correspondre à une volonté de refuser, au moins temporairement, le rôle domestique qui leur est alors consubstantiel; plus généralement, nombre de femmes contournent les consignes 
édictées par les hommes de leur entourage quant aux "bonnes" et "mauvaises" lectures; surtout, la lecture se pratique de façon fragmentaire, car le temps libre est lui-même parcellaire, ce qui peut favoriser une approche plus critique des livres, tout comme la discussion des œuvres lues par le biais de la correspondance entre quelques intimes. Toutefois, c'est ici que les analyses de Lyons rencontrent leurs limites, dont il est d'ailleurs lui-même parfaitement conscient. Il s'agit en premier lieu de la relative étroitesse du corpus utilisé (34 autobiographies, dont 8 seulement sont écrites par des femmes), qui permet de décrire certaines expériences mais qui rend hasardeux tout essai de généralisation, et, ensuite, du biais social qui est inévitable : les pratiques des femmes des milieux populaires sont bien plus difficiles à percevoir, et Lyons a ici largement recours aux travaux réalisés par Anne-Marie Thiesse dans les années 1980. La même difficulté apparaît aussi dans le chapitre consacré aux paysans, à la place bien sûr capitale dans la société française $\mathrm{du} \mathrm{XIX}^{\mathrm{e}}$ siècle. La réévaluation, à la fois quantitative et qualitative, de l'impact de la culture imprimée sur le monde paysan diffère sensiblement des conclusions d'Eugen Weber dans La Fin des terroirs, sans doute parce que son étude faisait la part trop belle aux régions les plus retardées de France. Régulièrement, et indépendamment de la nature du régime en place, les autorités s'inquiétèrent de la diffusion dans les campagnes de brochures politiquement subversives, et des mesures furent prises pour y répondre, que ce soit la lutte contre le colportage ou l'établissement de bibliothèques scolaires; toutefois, la rareté extrême des sources de première main rend difficile une approche véritablement nourrie de l'autre aspect de cette problématique centrale de l'ouvrage, à savoir les modalités de l'appropriation de l'imprimé par ses nouveaux lecteurs. Cependant, on ne peut que douter qu'il soit possible de surmonter ces obstacles et cela ne diminue en rien l'apport de Martyn Lyons, qui livre un travail très subtil sur un thème central pour l'histoire culturelle.

\section{NOTES}

1.. Martin LYONS, Le triomphe du livre. Une histoire sociologique de la lecture dans la France du XIXe siècle, Paris, Éditions Promodis, 1987. 